\title{
Probable Value for the Next Sunspot Minimum
}

\author{
Virginia Mabel Silbergleit ${ }^{1,2}$ \\ ${ }^{1}$ National Council of Scientific and Technological Research (CONICET), Argentina \\ ${ }^{2}$ Institute of Gas and Petroleum (IGPUBA), School of Engineering, University of Buenos Aires, Avenida Las Heras 2214, \\ C1056ACV, Argentina
}

Correspondence should be addressed to Virginia Mabel Silbergleit; vsilbergleit@fi.uba.ar

Received 4 October 2013; Revised 20 December 2013; Accepted 23 December 2013; Published 5 February 2014

Academic Editor: Gary Wegner

Copyright (c) 2014 Virginia Mabel Silbergleit. This is an open access article distributed under the Creative Commons Attribution License, which permits unrestricted use, distribution, and reproduction in any medium, provided the original work is properly cited.

Gumbel's first distribution is applied to smoothed monthly mean sunspot numbers for solar cycles 10 to 24 . According to that, the next minimum for solar cycle 24-25 transition would be the deepest solar minimum of the last 150 years. This study provides an additional insight about changes in the Sun which are currently happening.

\section{Introduction}

The sunspot cycle is characterized by its variability as much as its regularity. Sunspots are regions of strong photospheric magnetic fields that have the polarities north and south. The spots change with an approximate 11-year period and their magnetic cycle is twice as long. Physical processes contribute to produce the Sun's 11-year activity cycle as the occurrence of coronal holes. Many models of the solar dynamo exist but none is complete. Part of the solution related to flows in the solar convection zone is studied by helioseismology and large-scale numerical models. By using dynamo theory the cycle's activity is predicted with one parameter of physical analysis [1]

The Sun shows a roughly 11-year cycle of activity, from stormy to quiet cycles and back again (as it is shown in Figure 1).

The solar wind velocity and the strength and direction of the interplanetary magnetic field affect the coupling of energy from the solar wind into the magnetosphere. Some part of this energy is transitorily stored in the magnetosphere and then it is finally deposited in the upper atmosphere in auroral processes and current-driven heating. Properties of the solar wind and interplanetary magnetic field are correlated with the geomagnetic activity (see [2-4]).

The last solar minimum, currently ending solar cycle 23, is singular. Prior five solar minima occurred in 2008, 1996, 1986,
1976, and 1966. Current cycle had its minimum in December 2008.

The space missions are optimized to study solar maximum conditions and not solar minimum states. The proceedings of the twenty-third series of international workshops related to the $\mathrm{SOHO}$ mission show a discussion about if the Sun is at the beginning of a new grand minimum of activity, similar to the historical Dalton and Maunder minima.

The Dalton minimum (1795-1830), the Maunder minimum (1635-1705), and the Spörer minimum (1450-1550) happened when the Sun was quite different from now (e.g., [5]).

Deep solar minima appear randomly and the Sun gives back fast. Last solar minimum was one of the deep solar minima that appear every century [6].

Possibly, the solar minimum is related to the basic understanding of the solar dynamo and its effects on the photosphere and the corona.

According to a long-term analysis [7] it was estimated that the galactic cosmic ray aspect during the recent solar minimum was the largest of previous minima and the increase was more pronounced for locations outside the magnetosphere.

The expanded last solar minimum has produced even more novel conditions in the solar wind and in the Earth's magnetosphere when compared in opposition to the records obtained since the beginning of the space age. The observed solar wind value of $370 \mathrm{~km} \mathrm{~s}^{-1}$ is depressed in average value. 


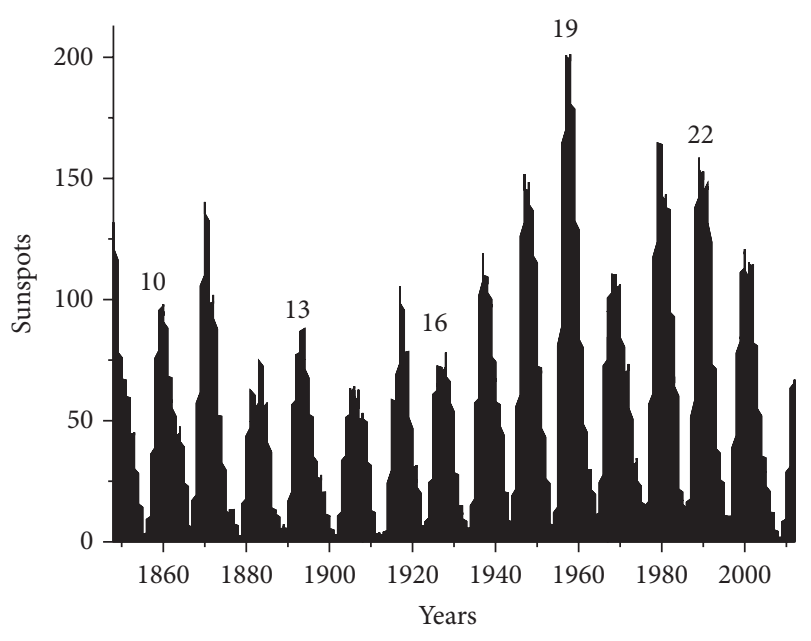

FIGURE 1: Spots for the last fifteen solar cycles. These data are well documented over the last centuries and show a 10-11-year pattern.

The predicted values such as the $300 \mathrm{~km} \mathrm{~s}^{-1}$ in 1912-1913 are not good enough to be compared with the cycle 23-24 values. The median speed of the solar wind is $450 \mathrm{~km} \mathrm{~s}^{-1}$ and consists of fast streams which rise from $350 \mathrm{~km} \mathrm{~s}^{-1}$ to $600 \mathrm{~km} \mathrm{~s}^{-1}$, and when these fast streams vanish, the radiation belts measured by SAMPEX rapidly decline in flux [6]. The last three solar cycles (1976-2008) had enough solar wind measurements to have last solar minimum with a near term perspective; these solar cycles had greater solar activity than normal. This period has been called a grand solar maximum (e.g., [8]).

The magnetic field production was weak in solar cycle 23 , and along solar cycles 21 and 22, strong polar fields were produced by this flux transport. The solar magnetic dynamo can change on decadal scales and should reveal how the observed solar field is generated. This behavior was not usual over the last 30-40 years because we did not have uniform records before 1970. The Sun is still behaving strangely in comparison with the last few solar minima [6].

By using the dynamo theory Schatten et al. [1] predicted the cycle's activity using one parameter of physical analysis.

By considering polar field measurements available for four solar cycles, Svalgaard et al. [9] predicted that the approaching solar cycle 24 will be potentially the smallest cycle in the last 100 years.

Duhau [10] presented evidence that solar activity is in a declining time from the year 1993.

Hathaway [11] studied international sunspot counts and found that the average period of a solar cycle is 131 months with a standard deviation of 14 months. Decaying solar cycle 23 has so far lasted 142 months and is not abnormally depressed. The available 13-smoothed monthly sunspot (MSSM) was 5.70. This is larger than 12 of the last 23 solar minimum values.

To explain changes in space weather, it is necessary to study data and their connections between the effects and the involved physics.

Before last solar minimum, the sunspots and records of solar activity manifested that these years were times of high solar wind; then the Sun will return to less active conditions and will do for a relatively long period of time, according to the fact that it is necessary to monitor carefully the polar regions of the Sun. However, there is much to be learned about the Sun, even at quiet times. There are magnetic regions appearing; there are coronal holes, fast and slow streams, and time variations in the solar wind. There are reasons to think that the low field intensities happened during last solar minimum presage of a low-activity cycle.

To analyze SMMS, Gumbel's asymptotic distributions [12] are considered. They were applied by Siscoe $[13,14]$ and Silbergleit et al. $[15,16]$ to study sunspots in each solar cycle and the largest geomagnetic storms. Analytic expressions are obtained for extreme value populations from which the statistical parameters-mode, median, mean, and standard deviation-are derived.

Therefore, it is important to review the sunspot series in the last centuries as a manner to evaluate changes in the Sun. Figure 1 shows the sunspot numbers related to the last fifteen solar cycles. To analyze the statistics of solar minima, the period from 1848 to 2013 is considered due to the goodness of the data (see [17]), because the sunspots records are considered unsatisfactory during 1700-1748, questionable during 1749-1817, and certain since 1848.

\section{Data and Prediction Technique}

The data (smoothed monthly mean sunspots, SMMS) were downloaded from $\mathrm{ftp}: / / \mathrm{ftp}$.ngdc.noaa.gov/, that is the web side of the National Geophysical Data Center.

Extreme value statistics are useful to study the tails of series (the lowest or highest values) for data normally distributed. While it is not often important to know the distribution of variables which describe a population, by studying their tails (that appear to be normally distributed) useful information about the distribution itself can be yielded (on the basis of extreme statistics [12]).

For a given minima observation, the probability that this value could be less than $s$ is $p=\beta(s)$, where Gumbel's first asymptotic distribution is described by $\beta(s)$. The probability that this value could be equal to or greater than $\mathrm{s}$ is $P=[1-$ $\beta(s)]$ (see [12]).

The theory of extremes gives the mathematical expression of $\beta(s)$ :

$$
\beta(s)=\exp \{-\exp [-(a+b s)]\},
$$

where $a$ and $b$ are constants obtained by a linear square fit.

As the probability function for the maximum amplitude for each solar cycle is unknown, the observed values of $\beta(s)$ are deduced according to [12]. For $N$ observed extreme values (where $N$ is the total of data), the relationship between $\beta(s)$ and $s$ is obtained.

Extreme magnitudes are calculated by considering the solar cycle data between the years 1848 and 2013 and by using the minimum sunspot numbers per cycle (s) as they are shown in Table 1.

In order to use all fifteen observations, a procedure useful for small samples was introduced by [18]. With the limited 
TABLE 1: Minima sunspots per solar cycle and the plotting values considered.

\begin{tabular}{lcccc}
\hline Order & Data & Probability & Plotting value & Cycle \\
\hline 1 & 1.50 & $3.7 E-02$ & -1.19 & 15 \\
2 & 1.70 & $1.03 E-01$ & $-8.2 E-01$ & 24 \\
3 & 2.20 & $1.69 E-01$ & $-5.7 E-01$ & 12 \\
4 & 2.60 & $2.35 E-01$ & $-3.7 E-01$ & 14 \\
5 & 3.20 & $3.02 E-01$ & $-1.8 E-01$ & 10 \\
6 & 3.40 & $3.68 E-01$ & $-4.2 E-04$ & 19 \\
7 & 3.40 & $4.34 E-01$ & $1.8 E-01$ & 17 \\
8 & 5.20 & $5.00 E-01$ & $3.7 E-01$ & 13 \\
9 & 5.20 & $5.66 E-01$ & $5.6 E-01$ & 11 \\
10 & 5.60 & $6.32 E-01$ & $7.8 E-01$ & 16 \\
11 & 7.70 & $6.98 E-01$ & 1.02 & 18 \\
12 & 8.80 & $7.65 E-01$ & 1.32 & 23 \\
13 & 9.60 & $8.31 E-01$ & 1.68 & 20 \\
14 & 12.20 & $8.97 E-01$ & 2.22 & 21 \\
15 & 12.30 & $9.63 E-01$ & 3.28 & 22 \\
\hline
\end{tabular}

objective, a plotting rule [19] for the $i$ th of $N$ ordered data from a double exponential distribution is obtained as

$$
P_{i}=\frac{i-0.44}{N+0.12},
$$

where $N=15$ is the number of observations, $i$ is the order of the observation from the minima, and $P_{i}$ is the estimate of the cumulative frequency of the $i$ th term [19]. The related $P_{v i}$ values are defined by

$$
P_{v i}=-\ln \left[-\ln \left(P_{i}\right)\right] .
$$

The $P_{i}$ and $P_{v i}$ values are tabulated in columns 3 and 4 of Table 1 .

Figure 2 shows the straight line which represents the best fitting adjustment. The slope of the regression line is equal to $0.32 \pm 0.02$ in units of $\mathrm{SMMS}^{-1}$.

The return periods $T(s)$ and $t(s)$ are calculated by using the expressions:

$$
\begin{gathered}
T(s)=[1-\beta(s)]^{-1}, \\
t(s)=T(s)[T(s)-1]^{-1} .
\end{gathered}
$$

Equations (4) and (5) define $T(s)$ and $t(s)$. These values are plotted in Figure 3. $T(s)$ represents the expected times required to find one solar cycle minimum amplitude with the extreme equal to or exceeding abscissa ( $T$ branch); $t(s)$ shows the expected periods to have one cycle with extreme less than abscissa ( $t$ branch). These functions also show the increase in the expected range with a growing number of cycles. $T(s)$ and $t(s)$ mean that, for a given number of solar cycles (e.g., $M$ ), it is expected that $M-2$ values will be confined to a bounded interval. The bounds are estimated by (4) and (5), being one case higher (or lower) than the bound obtained through (4) (or (5)). For $M=2$ both bounds coincide at the median value (mv) which is the midpoint of the distribution (as it is shown in Figure 3).

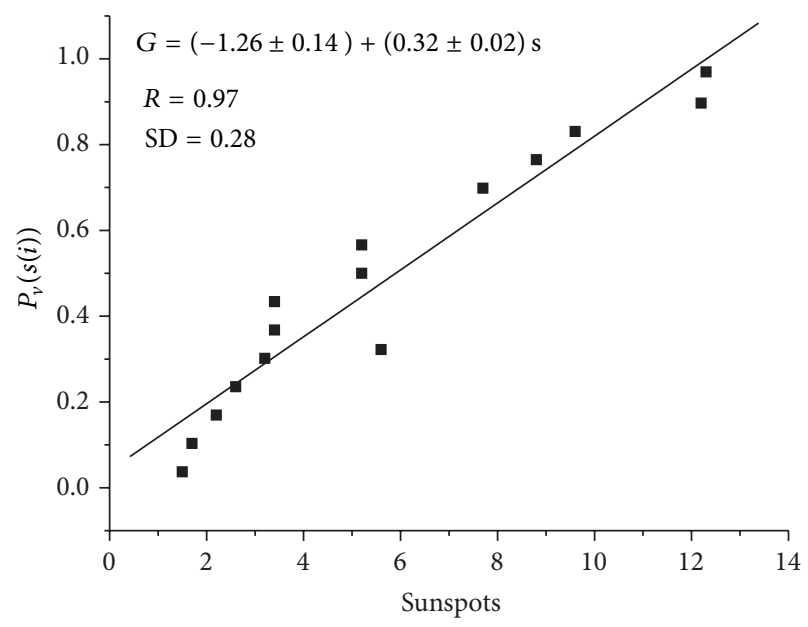

FIgURE 2: Data are plotted by using the ordinate values equal to $-\ln \left[-\ln \left(P_{i}\right)\right]$; they are indicated by squares. The terms $R$ and $\mathrm{SD}$ refer to the coefficient of correlation and the average error, respectively. $G$ is the inferred regression equation.

According to this theory, for sixteen cycles fourteen of them are expected to be in the range $12.3>s \geq 0.6$, one case lower than the lowest bound and the other one equal to or greater than the highest bound. In reference to Table 1, fourteen solar minima remain within the interval 12.3-0.6; one of them is equal to the highest bound (i.e., 12.3); then, the unknown next minimum should have a value lower than the lowest bound (i.e., 0.6).

As regards [13] the arithmetic mean (am), which is the average of all results, is related to the standard deviation $\mathrm{Sd}$ defined by [13] as $\mathrm{Sd}=1.2825 \mathrm{a}$.

The measure of the relative dispersion $\mathrm{Rd}$ is obtained to divide Sd by the mode. The statistical characteristic parameters of extreme values obtained are the mode $(\mathrm{mo}=$ $a / b=3.9)$, the median $(\mathrm{mv}=5.2)$, and the mean $(\mathrm{am}=5.6)$, all in units of SMMS with $\mathrm{Sd}=4.0 \mathrm{SMMS}$ and $\mathrm{Rd}=1.0$.

\section{Results, Discussion, and Conclusion}

Figure 2 shows the best fitting adjustment obtained considering Gumbel's first asymptotic distribution. The values of the parameters in (1) are $a=(-1.26 \pm 0.14)$ and $b=(0.32 \pm$ 0.02) SMMS.

The statistical characteristic parameters of extreme values obtained are $\mathrm{mo}=3.9$ sunspots, $\mathrm{mv}=5.2$, and $\mathrm{am}=$ 5.6 SMMS, with $\mathrm{Sd}=4.0$ SMMS and $\mathrm{Rd}=1.0$.

Figure 3 shows the return periods versus SMMS. The upper and lower bounds are shown for 16 solar cycles. According to that, fourteen minima are in the interval defined by 0.6 and 12.3 SMMS. One data (in Table 1 data number 15 for solar cycle 22) is on the upper bound; then it is expected that the next sunspot minimum would have a lower value than the lowest bound.

According to our results, the solar cycle 25 minimum could probably be the smallest of the last fifteen cycles. This result gives evidence of sunspots decrease and it agrees with 


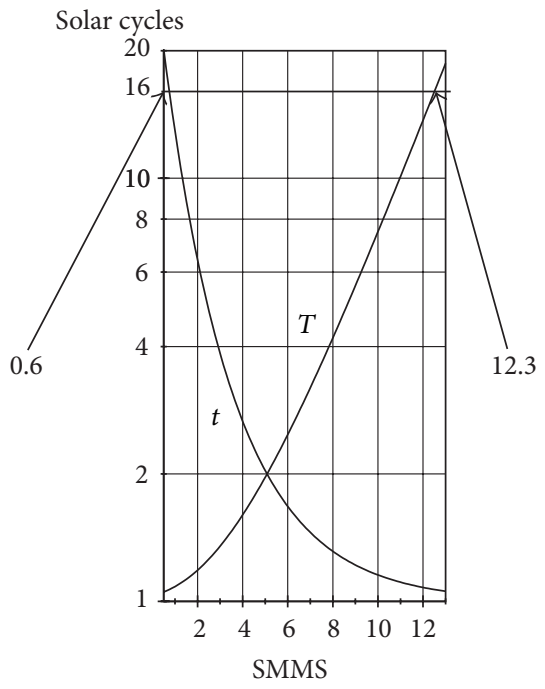

FIGURE 3: The ascending branch shows the expected number of periods required to detect one with extreme equal to or exceeding $s$. The descending one exhibits the expected number of intervals necessary to obtain one period with the extreme value less than $s$.

prior articles written by Kane [17], Svalgaard et al. [9], and Duhau [10] who predicted that solar activity is in a declining time and the current solar cycle will be the smallest cycle in the last 100 years. Sunspot numbers and every other indicator of solar activity continued depressed, as they did along 2008 and through mid-2009. According to the present study, the solar cycle 25 minimum would be almost a $60 \%$ smaller than the deeper cycle detected before (see Table 1). Thus, we conclude that we should be near the deepest minimum (as measured by smoothed monthly mean sunspots), if the statistics were the same as those for the last fifteen cycles.

\section{Conflict of Interests}

The author declares that there is no conflict of interests regarding the publication of this paper.

\section{Acknowledgments}

Thanks are due to the unknown reviewers for their valuable suggestions and comments. This research was partially supported by PIP $\mathrm{N}^{\circ} 11420090100258$ (CONICET) and PIP $\mathrm{N}^{\circ}$ 20020090100131 (UBACYT), Argentina.

\section{References}

[1] K. H. Schatten, P. H. Scherrer, L. Svalgaard, and J. M. Wilcox, "Using dynamo theory to predict the sunspot number in solar cycle 21," Geophysical Research Letters, vol. 5, no. 5, pp. 411-414, 1978.

[2] R. L. Arnoldy, "Signature in the interplanetary medium for substorms," Journal of Geophysical Research, vol. 76, no. 22, pp. 5189-5201, 1971.

[3] J. Hirshberg and D. S. Colburn, "Interplanetary field and geomagnetic variations-a unifield view," Planetary and Space Science, vol. 17, no. 6, pp. 1183-1206, 1969.
[4] C. T. Russell and R. L. McPherron, "Semiannual variation of geomagnetic activity," Journal of Geophysical Research, vol. 78, no. 1, pp. 92-108, 1973.

[5] J. A. Eddy, "Climate and the role of the sun, climate and history," in Studies in Interdisciplinary History, R. I. Rotberg and T. K. Rabb, Eds., p. 145, Princeton University Press, Princeton, NJ, USA, 1981.

[6] C. T. Russell, J. G. Luhmann, and L. K. Jian, "How unprecedented a solar minimum?" Reviews of Geophysics, vol. 48, no. 2, Article ID RG2004, 2010.

[7] A. I. Mrigakshi, D. Matthiä, T. Berger, G. Reitz, and G. WimmerSchweingruber, "Estimation of galactic cosmic ray exposure inside and outside the Earth's magnetosphere during the recent solar minimum between solar cycles 23 and 24," Advances in Space Research, vol. 52, no. 5, pp. 979-987, 2013.

[8] M. Lockwood, A. P. Rouillard, and I. D. Finch, "The rise and fall of open solar flux during the current grand solar maximum," Astrophysical Journal Letters, vol. 700, no. 2, pp. 937-944, 2009.

[9] L. Svalgaard, E. W. Cliver, and Y. Kamide, "Sunspot cycle 24: smallest cycle in 100 years?" Geophysical Research Letters, vol. 32, no. 1, Article ID L01104, 2005.

[10] S. Duhau, "An early prediction of maximum sunspot number in solar cycle 24," Solar Physics, vol. 213, no. 1, pp. 203-212, 2003.

[11] D. Hathaway, 2012, http://sciencel.nasa.gov/science-news/ science-at-nasa/2008/11jul_solarcycleupdate/.

[12] E. J. Gumbel, "On the frequency distribution of extreme values in meteorological data," Bulletin of the American Meteorological Society, vol. 23, pp. 95-104, 1942.

[13] G. L. Siscoe, "On the statistics of the largest sunspot number per solar cycle," Journal of Geophysical Research, vol. 81, pp. 62246226, 1976.

[14] G. L. Siscoe, "On the statistics of the largest geomagnetic storms per solar cycle," Journal of Geophysical Research, vol. 81, no. 25, pp. 4782-4784, 1976.

[15] V. M. Silbergleit, "On the statistics of maximum sunspot numbers," Journal of Atmospheric and Solar-Terrestrial Physics, vol. 60, no. 17, pp. 1701-1710, 1998.

[16] V. M. Silbergleit and P. A. Larocca, "Geomagnetic activity and solar cycles," Advances in Space Research, vol. 36, no. 12, pp. 2384-2387, 2005.

[17] R. P. Kane, "Prediction of solar cycle maximum using solar cycle lengths," Solar Physics, vol. 248, no. 1, pp. 203-209, 2008.

[18] W. C. Krumbein and J. Lieblein, "Geological application of extreme-value methods to interpretation of cobbles and boulders in gravel deposits," Transactions, American Geophysical Union, vol. 37, pp. 313-319, 1955.

[19] I. Gringorten, "A plotting rule for extreme probability paper," Journal of Geophysical Research, vol. 68, no. 3, pp. 813-814, 1963. 

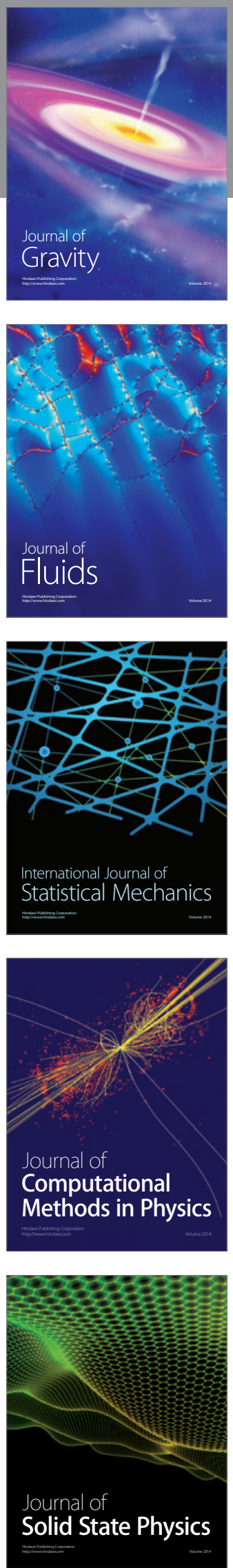

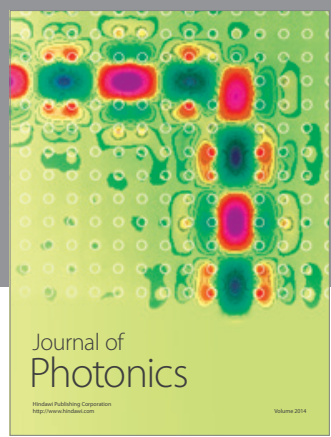

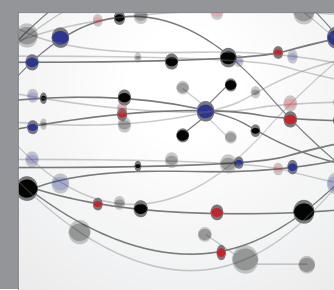

The Scientific World Journal

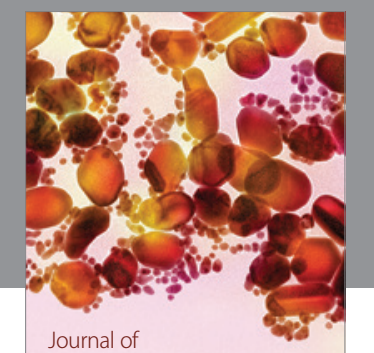

Soft Matter
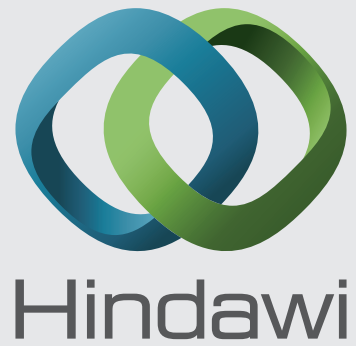

Submit your manuscripts at

http://www.hindawi.com
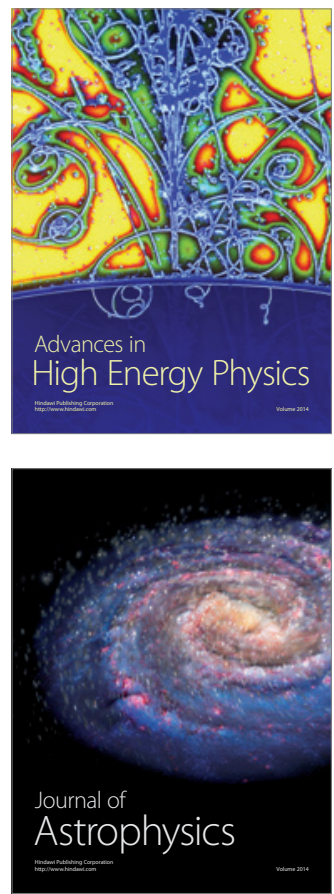
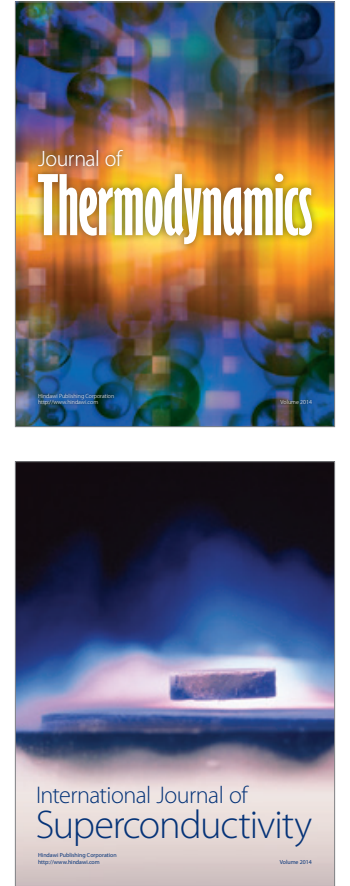
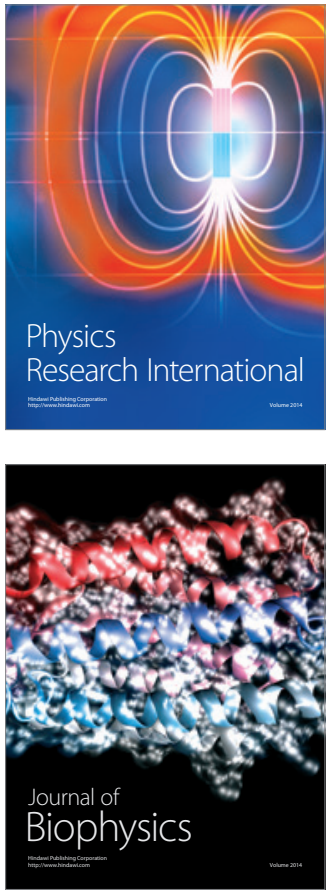
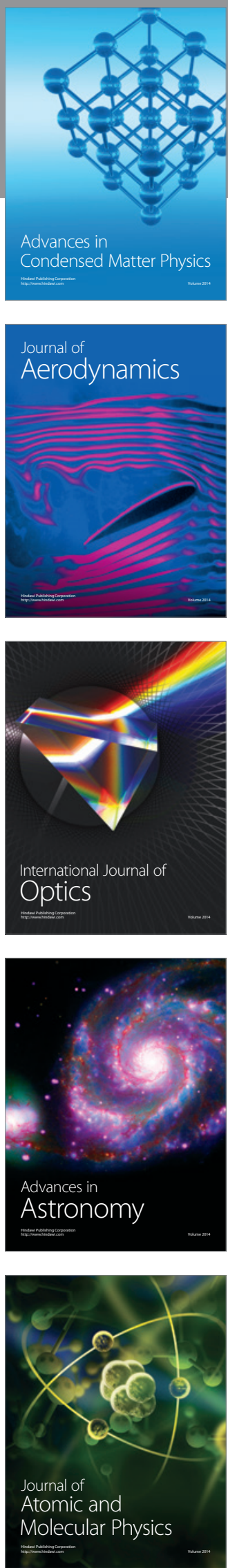